\title{
GEOMETRIC SINGULAR PERTURBATION THEORY FOR NON-SMOOTH DYNAMICAL SYSTEMS
}

\author{
Pedro T. Cardin, Paulo R. da Silva, and Marco A. Teixeira
}

Abstract: In this article we deal with singularly perturbed Filippov systems $Z_{\varepsilon}$ :

$$
\dot{x}=\left\{\begin{array}{ll}
F(x, y, \varepsilon) & \text { if } h(x, y, \varepsilon) \leq 0, \\
G(x, y, \varepsilon) & \text { if } h(x, y, \varepsilon) \geq 0,
\end{array} \quad \varepsilon \dot{y}=H(x, y, \varepsilon),\right.
$$

where $\varepsilon \in \mathbb{R}$ is a small parameter, $x \in \mathbb{R}^{n}, n \geq 2$, and $y \in \mathbb{R}$ denote the slow and fast variables, respectively, and $F, G, h$, and $H$ are smooth maps. We study the effect of singular perturbations at typical singularities of $Z_{0}$. Special attention will be dedicated to those points satisfying $q \in\{h(x, y, 0)=0\} \cap\{H(x, y, 0)=0\}$ where $F$ or $G$ is tangent to $\{h(x, y, 0)=0\}$. The persistence and the stability properties of those objects are investigated.

2010 Mathematics Subject Classification: Primary: 34C20, 34C26, 34D15, $34 \mathrm{H} 05$.

Key words: Filippov systems, singular perturbation, tangency points.

\section{Introduction}

Non-smooth dynamical systems (or Filippov systems) occur in various situations like mechanical systems with dry friction or with impacts. They also appear in control theory, electronic, economics, medicine and biology (see for instance $[\mathbf{1}],[\mathbf{3}],[\mathbf{6}]$ and $[\mathbf{7}]$ ). All these motivational models emerge from differential equations with discontinuous right-hand sides.

Let $U \subset \mathbb{R}^{n}$ be an open set. We denote by $C^{r}\left(U, \mathbb{R}^{n}\right)$ the set of all vector fields of class $C^{r}$ defined on $U$, with $r \geq 1$, endowed with the $C^{r}$-topology.

The simplest case of a Filippov system occurs when the phase space is composed by two domains such that for each domain a different ODE (ordinary differential equation) governs the dynamics, namely

$$
\dot{x}=Z(x)= \begin{cases}F(x) & \text { if } h(x) \leq 0, \\ G(x) & \text { if } h(x) \geq 0 .\end{cases}
$$


In equation (2), $F, G \in C^{r}\left(U, \mathbb{R}^{n}\right)$ and $h: U \rightarrow \mathbb{R}$ is a smooth function having $0 \in \mathbb{R}$ as a regular value. The common boundary $\mathcal{M}=\{x \in$ $U \mid h(x)=0\}$ between the domains $\mathcal{M}_{-}=\{x \in U \mid h(x) \leq 0\}$ and $\mathcal{M}_{+}=\{x \in U \mid h(x) \geq 0\}$ is called the switching manifold. We will use the notation $Z=(F, G)$ to represent the Filippov system (2) and denote by $\Omega^{r}(U)$ the set of all vector fields $Z$ of the form (2) defined on $U$.

We also use $F h(p)=F(p) \cdot \nabla h(p)$ to denote the scalar product in $\mathbb{R}^{n}$ between the vector field $F: U \rightarrow \mathbb{R}^{n}$ and the gradient of the function $h: U \rightarrow \mathbb{R}$. For $k \geq 2$, we define inductively $F^{k} h(p)=F\left(F^{k-1} h\right)(p)$.

On the switching manifold $\mathcal{M}$ the following open sets are distinguished:

- Sewing region: $\mathcal{M}^{1}=\{p \in \mathcal{M}:[F h(p)][G h(p)]>0\}$.

- Escaping region: $\mathcal{M}^{2}=\{p \in \mathcal{M}: F h(p)<0, G h(p)>0\}$.

- Sliding region: $\mathcal{M}^{3}=\{p \in \mathcal{M}: F h(p)>0, G h(p)<0\}$.

The definitions of these three regions exclude the so-called tangency points, that is, points where one of the two vector fields $F$ or $G$ is tangent to $\mathcal{M}$. They are characterized by $p \in \mathcal{M}$ such that $F h(p)=0$ or $G h(p)=0$. Generically speaking, these points are on the boundary of the regions $\mathcal{M}^{1}, \mathcal{M}^{2}$, and $\mathcal{M}^{3}$, which we denote by $\partial \mathcal{M}^{1}, \partial \mathcal{M}^{2}$, and $\partial \mathcal{M}^{3}$, respectively. Tangency points include the case $F(p)=0$ or $G(p)=0$, that is, when one of the two vector fields has an equilibrium point at $\mathcal{M}$. We define two types of tangency between a smooth vector field and a manifold, which will be used in the paper.

Definition 1.1. We say that a smooth vector field $F$ has a fold or quadratic tangency with $\mathcal{M}=\{h(x)=0\}$ at a point $p \in \mathcal{M}$ provided $F h(p)=0$ and $F^{2} h(p) \neq 0$.

Definition 1.2. A smooth vector field $F$ has a cusp or cubic tangency with $\mathcal{M}=\{h(x)=0\}$ at a point $p \in \mathcal{M}$ provided $F h(p)=F^{2} h(p)=$ $0, F^{3} h(p) \neq 0$, and the set $\left\{\nabla h(p), \nabla(F h)(p), \nabla\left(F^{2} h\right)(p)\right\}$ is linearly independent.

If a point of the phase space which is moving on an orbit of $Z=$ $(F, G)$ falls onto $\mathcal{M}^{1}$ then it crosses $\mathcal{M}^{1}$ to another part of the space. In $\mathcal{M}^{2}$ and $\mathcal{M}^{3}$, the definition of the local orbit is given by the Filippov convention $[\mathbf{5}]$. We consider the vector field $Z^{S}$ which is the linear convex combination of $F$ and $G$ tangent to $\mathcal{M}$, that is

$$
\dot{x}=Z^{S}(x)=\frac{[\nabla h(x) F(x)] G(x)-[\nabla h(x) G(x)] F(x)}{\nabla h(x)[F(x)-G(x)]} .
$$


We call $Z^{S}$ the sliding vector field associated to the Filippov system (2), independently on whether it is defined in the sliding or escaping regions (see Figure 1). Solutions of $Z=(F, G)$ through points of $\mathcal{M}^{2} \cup \mathcal{M}^{3}$ follow the orbit of $Z^{S}$.

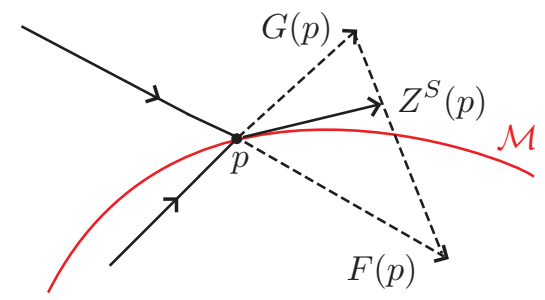

Figure 1. The sliding vector field $Z^{S}$.

The singularities of a Filippov vector field (2) are

- $p \in \mathcal{M}_{ \pm}$such that $p$ is an equilibrium of $F$ or $G$, that is, $F(p)=0$ or $G(p)=0$, respectively;

- $p \in \mathcal{M}^{2} \cup \mathcal{M}^{3}$ such that $p$ is an equilibrium of $Z^{S}$, that is, $Z^{S}(p)=0$;

- $p \in \partial \mathcal{M}^{1} \cup \partial \mathcal{M}^{2} \cup \partial \mathcal{M}^{3}$, that is, the tangency points $(F h(p)=0$ or $G h(p)=0)$.

Fenichel (see $[\mathbf{4}, \mathbf{9}]$ ) proved that, in smooth dynamical systems, any structure which persists under regular perturbation also persists under singular perturbation. In [2] we extended this theory for the sliding vector field associated to the reduced problem of (1), that is, we study how the sliding mode in Filippov systems is affected by singular perturbations. In this paper we consider again systems of the form (1). Now we analyze the effect of singular perturbations at the tangency points.

For each $\varepsilon \geq 0$ we denote by $\mathcal{M}_{\varepsilon}$ the set $\mathcal{M}_{\varepsilon}=\{h(x, y, \varepsilon)=0\}$. Note that $\mathcal{M}_{\varepsilon}$ is the switching manifold of (1). The set $\mathcal{S}_{0}:=\{H(x, y, 0)=$ $0\}$ is called the critical manifold of the singular perturbation problem (1). For $\varepsilon>0$, we also use the notation $\mathcal{N}_{\varepsilon}$ to denote the set $\mathcal{N}_{\varepsilon}=\{H(x, y, \varepsilon)=0\}$. Here we are supposing that $\mathcal{M}_{0}$ and $\mathcal{S}_{0}$ are in a general position, i.e., $\nabla h(p)$ and $\nabla H(p)$ are linearly independent for any $p \in \mathcal{M}_{0} \cap \mathcal{S}_{0}$. Figure 2 illustrates when the manifolds $\mathcal{M}_{0}$ and $\mathcal{S}_{0}$ are in a general position and their $\varepsilon$-perturbations $\mathcal{M}_{\varepsilon}$ and $\mathcal{N}_{\varepsilon}$. Throughout this article we will assume that the equation $H(x, y, \varepsilon)=0$ can be solved by $y=f_{\varepsilon}(x)$, for all $\varepsilon \geq 0$. 


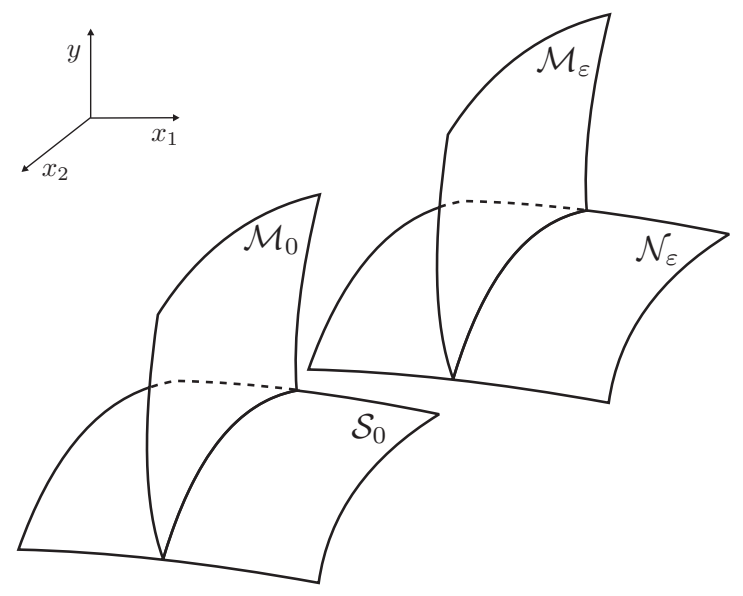

Figure 2. Manifolds $\mathcal{M}_{0}$ and $\mathcal{S}_{0}$ in a general position and their $\varepsilon$-perturbations $\mathcal{M}_{\varepsilon}$ and $\mathcal{N}_{\varepsilon}$.

For $\varepsilon=0$ in (1) we have the so-called reduced problem

$$
\dot{x}=\left\{\begin{array}{ll}
\widetilde{F}(x) & \text { if } \widetilde{h}(x) \leq 0, \\
\widetilde{G}(x) & \text { if } \widetilde{h}(x) \geq 0,
\end{array} \quad 0=\widetilde{H}(x),\right.
$$

where $\widetilde{F}(x)=F\left(x, f_{0}(x), 0\right), \widetilde{G}(x)=G\left(x, f_{0}(x), 0\right), \widetilde{h}(x)=h\left(x, f_{0}(x), 0\right)$ and $\widetilde{H}(x)=H\left(x, f_{0}(x), 0\right)$. The reduced problem (4) is a dynamical system defined on the manifold $\mathcal{S}_{0}$. For $\varepsilon \neq 0$ we can write the systems of the form (1) in the general form given in (2), namely

$$
(\dot{x}, \dot{y})= \begin{cases}\bar{F}(x, y, \varepsilon) & \text { if } h(x, y, \varepsilon) \leq 0, \\ \bar{G}(x, y, \varepsilon) & \text { if } h(x, y, \varepsilon) \geq 0,\end{cases}
$$

where $\bar{F}(x, y, \varepsilon)=(F, H / \varepsilon)$ and $\bar{G}(x, y, \varepsilon)=(G, H / \varepsilon)$. We will use the notation $Z_{\varepsilon}=(\bar{F}, \bar{G})$ to represent the Filippov slow-fast system (5) and $Z_{0}$ to represent the reduced problem (4).

Definition 1.3. We say that a system of the form (1) is locally simple at $p=\left(x_{0}, y_{0}, 0\right) \in \mathbb{R}^{n+1} \times \mathbb{R}$ if one of the following conditions is satisfied:

(i) $\frac{\partial h}{\partial x}(p) \neq 0$ and $h\left(x_{0}, y, 0\right)=0$, for all $y$ close to $y_{0}$; or

(ii) there exists a neighborhood $U$ of $\left(x_{0}, y_{0}\right)$ in $\mathbb{R}^{n+1}$ such that $\frac{\partial H}{\partial x}(q)=$ 0 , for all $q \in U \cap \mathcal{M}_{\varepsilon}$.

Remark 1. Note that for the case (i) of Definition 1.3, we can choose local coordinates (around the point $p$ ) such that $h(x, y, \varepsilon)=\bar{h}(x, \varepsilon)$, i.e., 
the function $h$ does not depend on the variable $y$ in a given neighborhood of $p$.

In what follows we summarize a rough overall description of the main results of the paper. The precise statements are given in Sections 2, 3 and 4 . In all of them, we assume that the systems of the form (1) are locally simple at the singularity.

First result (Theorem A): This result concerns singular perturbations of fold-regular singularities (see Definition 2.1) of the reduced problem (4). Assuming that system (4) has a singularity of fold-regular type $\bar{p}$, we state that, for $\varepsilon \neq 0$ sufficiently small, there is a family $\bar{p}_{\varepsilon}$ of fold-regular singularities for the corresponding system (1) such that $\bar{p}_{0}=\bar{p}$. Moreover, the features of $\bar{p}$ are preserved for $\bar{p}_{\varepsilon}$.

Second result (Theorem B): We study singular perturbations of fold-fold singularities (see Definition 2.2) of the reduced problem (4) with $n \geq 3$. If system (4) has a singularity of fold-fold type $\bar{p}$ then, for $\varepsilon \neq 0$ sufficiently small, there is a family $\bar{p}_{\varepsilon}$ of fold-fold singularities for the corresponding system (1) such that $\bar{p}_{0}=\bar{p}$. Moreover, the features of $\bar{p}$ are preserved for $\bar{p}_{\varepsilon}$.

Third result (Theorem $\mathbf{C}$ ): This result concerns singular perturbations of cusp-regular singularities (see Definition 3.1) of system (4) with $n=2$. We prove that the persistence (or not) of tangency points is determined by the sign of a continuous function depending of $\varepsilon$ sufficiently small.

Fourth result (Theorem D): This result concerns singular perturbations of fold-cusp singularities of the reduced problem (4) with $n \geq 4$. Supposing that system (4) has a fold-cusp singularity $\bar{p}$, we prove that, for $\varepsilon \neq 0$ sufficiently small, there is a family $\bar{p}_{\varepsilon}$ of fold-cusp singularities for the corresponding system (1) such that $\bar{p}_{0}=\bar{p}$.

Fifth result (Theorem E): We study singular perturbations of hyperbolic/equilibrium-regular singularities (see Definition 4.1) of (4) with $n=2$. If system (4) has a singularity of hyperbolic/equilibrium-regular type $\bar{p}$ then there is a neighborhood $W$ of $\bar{p}$ such that system (1), for $\varepsilon \neq 0$ sufficiently small, has a hyperbolic equilibrium $\bar{p}_{\varepsilon} \in W$. Moreover, if $\bar{p}_{\varepsilon} \notin \mathcal{M}_{\varepsilon}$, then there exists a family $\left\{\bar{q}_{\varepsilon}\right\}$ of fold-regular singularities of (1).

The paper is organized as follows. In Section 2 we study the effect of singular perturbations at fold points. We state that fold singularities 
are persistent with respect to singular perturbations (see Theorems A and B). In Section 3 we study the effect of singular perturbations at cusp points in dimension two. We show, with a minimal example, that such kind of singularities are not persistent with respect to singular perturbations. In this section we also study the unfolding of cusp singularities (see Theorem C). In Subsection 3.1, fold-cusp singularities are considered (see Theorem D). In Section 4 we study the effect of singular perturbation at hyperbolic points in dimension two (see Theorem E). The proofs of the main results are in Section 5. Final remarks are presented in Section 6.

\section{Singular perturbation of fold singularities}

In this section we will study the effect of singular perturbations at fold singularities of the reduced problem (4). More precisely, by assuming that the reduced problem (4) has fold singularities, we want to know if they persist for the full system (5), for $\varepsilon \neq 0$ small. We also present an example in dimension two showing that in general fold-fold singularities are not persistent with respect to singular perturbations. On the other hand, generically speaking, they are persistent in dimension three (see Theorem B).

Definition 2.1. We say that $p \in \mathcal{M}$ is a fold-regular singularity of (2) if

(i) $F h(p)=0, F^{2} h(p) \neq 0$, and $G h(p) \neq 0$; or

(ii) $G h(p)=0, G^{2} h(p) \neq 0$, and $F h(p) \neq 0$.

In the first case, we say that the fold-regular singularity $p \in \mathcal{M}$ is visible if $F^{2} h(p)<0$ and invisible if $F^{2} h(p)>0$. In the second case, it is visible provided $G^{2} h(p)>0$ and invisible provided $G^{2} h(p)<0$.

Definition 2.2. Let $p \in \mathcal{M}$ be a fold-fold singularity of (2), i.e., both vector fields $F$ and $G$ have a fold or quadratic tangency at the same point $p \in \mathcal{M}$. We distinguish the following cases:

(i) Elliptic case: $F^{2} h(p)>0$ and $G^{2} h(p)<0$ (invisible two-fold).

(ii) Parabolic case: $F^{2} h(p)<0$ and $G^{2} h(p)<0$ (visible fold-invisible fold) or $F^{2} h(p)>0$ and $G^{2} h(p)>0$ (invisible fold-visible fold).

(iii) Hyperbolic case: $F^{2} h(p)<0$ and $G^{2} h(p)>0$ (visible two-fold).

Remark 2. We define fold-regular and fold-fold singularities for system (4) similarly to what was done in Definitions 2.1 and 2.2, respectively. We only note that system (4) is defined on the manifold $\mathcal{S}_{0}=$ $\{H(x, y, 0)=0\}$. 
In the theorem below we will see that fold-regular singularities are robust with respect to singular perturbations.

Theorem A. Let $Z_{\varepsilon}(x, y)$ be a $C^{r}$ family defined by (1), with $r \geq 2$. Consider $\bar{p}=\left(p, f_{0}(p), 0\right) \in \mathcal{S}_{0} \cap \mathcal{M}_{0}$ a fold-regular singularity of the reduced problem $Z_{0}$ and suppose that $Z_{\varepsilon}$ is locally simple at $\bar{p}$. Then there exists $\varepsilon_{1}>0$ such that:

(i) There is a $C^{r-1}$ family $\left\{\bar{p}_{\varepsilon}: \varepsilon \in\left(-\varepsilon_{1}, \varepsilon_{1}\right)\right\}$ such that $\bar{p}_{0}=\bar{p}$ and $\bar{p}_{\varepsilon}$ is a fold-regular singularity of $Z_{\varepsilon}$.

(ii) If $\bar{p}$ is a visible (resp. invisible) fold-regular of $Z_{0}$ then $\bar{p}_{\varepsilon}$ is a visible (resp. invisible) fold-regular of $Z_{\varepsilon}$.

Figure 3 illustrates the case where the reduced problem $Z_{0}$ has a visible fold-regular singularity $\bar{p}$. If $Z_{\varepsilon}$ is locally simple at $\bar{p}$, Theorem A ensures that $Z_{\varepsilon}$ has a visible fold-regular singularity $\bar{p}_{\varepsilon}$, for all $\varepsilon$ sufficiently small. Moreover, $\bar{p}_{\varepsilon} \rightarrow \bar{p}$ when $\varepsilon \rightarrow 0$. Theorem A is proved in Section 5. It is illustrated by the example below.

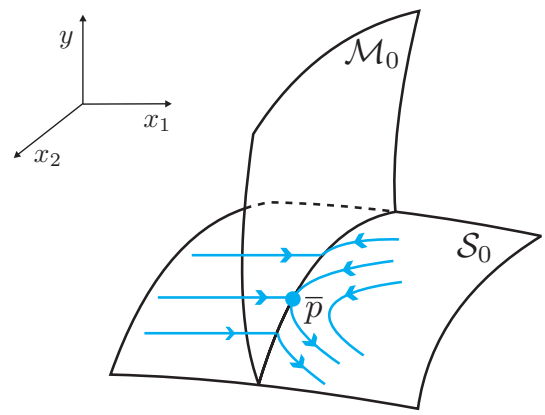

Figure 3. A visible fold-regular singularity $\bar{p}$ of the reduced problem $Z_{0}$.

Example 1. Consider the singularly perturbed Filippov system

$$
\dot{x}=\left\{\begin{array}{ll}
\left(x_{2}+y+\varepsilon, 1+\varepsilon\right) & \text { if } x_{1}-y-\varepsilon \leq 0, \\
(-1-\varepsilon,-1-\varepsilon) & \text { if } x_{1}-y-\varepsilon \geq 0,
\end{array} \quad \varepsilon \dot{y}=y-\varepsilon,\right.
$$

where $x=\left(x_{1}, x_{2}\right) \in \mathbb{R}^{2}$. The functions $h(x, y, \varepsilon)$ and $H(x, y, \varepsilon)$ that define the manifolds $\mathcal{M}_{\varepsilon}$ and $\mathcal{N}_{\varepsilon}$ are given by $h(x, y, \varepsilon)=x_{1}-y-\varepsilon$ and $H(x, y, \varepsilon)=y-\varepsilon$. The critical manifold is $\mathcal{S}_{0}=\{y=0\}$. For $\varepsilon=0$ 
in (6) we have the reduced problem

$$
\dot{x}=\left(\dot{x}_{1}, \dot{x}_{2}\right)=\left\{\begin{array}{ll}
\left(x_{2}, 1\right) & \text { if } x_{1} \leq 0, \\
(-1,-1) & \text { if } x_{1} \geq 0
\end{array} \quad y=0\right.
$$

By denoting system (7) as in (4), we have that

$$
\widetilde{F} \tilde{h}=x_{2}, \quad \widetilde{F}^{2} \widetilde{h}=1, \quad \widetilde{G} \widetilde{h}=-1 .
$$

Thus, $\bar{p}=(0,0)$ is an invisible fold-regular singularity of $(7)$. Now, let us write (6) in the general form given by (5), where

$$
\bar{F}(x, y, \varepsilon)=\left(x_{2}+y+\varepsilon, 1+\varepsilon, \frac{y}{\varepsilon}-1\right)
$$

and

$$
\bar{G}(x, y, \varepsilon)=\left(-1-\varepsilon,-1-\varepsilon, \frac{y}{\varepsilon}-1\right) .
$$

We have that

$$
\bar{F} h=x_{2}+y-\frac{y}{\varepsilon}+\varepsilon+1, \quad \bar{F}^{2} h=\frac{y}{\varepsilon}-\frac{y}{\varepsilon^{2}}+\frac{1}{\varepsilon}+\varepsilon, \quad \bar{G} h=-\frac{y}{\varepsilon}-\varepsilon .
$$

By solving the system $\bar{F} h\left(x, f_{\varepsilon}(x), \varepsilon\right)=0$ we obtain the $\varepsilon$-family $\bar{p}_{\varepsilon}=$ $(2 \varepsilon,-2 \varepsilon, \varepsilon)$. Since $\bar{F}^{2} h\left(\bar{p}_{\varepsilon}\right)=1+\varepsilon$ and $\bar{G} h\left(\bar{p}_{\varepsilon}\right)=-1-\varepsilon$, it follows that $\bar{p}_{\varepsilon}$ is an invisible fold-regular singularity of $(6)$, for all $\varepsilon \neq 0$ small.

For systems of the form (1) which are not locally simple at $\bar{p}$, we cannot guarantee the validity of the statement (ii) of Theorem A.

Counter-example 1. Consider the Filippov slow-fast system in $\mathbb{R}^{3}$

$$
\left(\dot{x}_{1}, \dot{x}_{2}\right)=\left\{\begin{array}{ll}
\left(x_{2}, 1\right) & \text { if } \theta x_{1}+(1-\theta) y \leq 0, \\
(-1,-1) & \text { if } \theta x_{1}+(1-\theta) y \geq 0,
\end{array} \quad \varepsilon \dot{y}=x_{1}-y\right.
$$

where $\theta$ is a real parameter, $\theta \neq 0$ and 1 . For $\varepsilon=0$ in (8) we have the reduced problem

$$
\left(\dot{x}_{1}, \dot{x}_{2}\right)=\left\{\begin{array}{ll}
\left(x_{2}, 1\right) & \text { if } x_{1} \leq 0, \\
(-1,-1) & \text { if } x_{1} \geq 0
\end{array} \quad x_{1}=y\right.
$$

It is easy to see that $(0,0)$ is an invisible fold-regular singularity of $(9)$. Note that system $(8)$ is not locally simple at $(0,0)$. 
By denoting system $(8)$ as in $(5)$, for $\varepsilon \neq 0$ we have

$$
\begin{aligned}
& \bar{F} h=\theta x_{2}+\frac{1-\theta}{\varepsilon}\left(x_{1}-y\right), \\
& \bar{F}^{2} h=\frac{1-\theta}{\varepsilon} x_{2}+\theta+\frac{\theta-1}{\varepsilon^{2}}\left(x_{1}-y\right), \\
& \bar{G} h=-\theta+\frac{1-\theta}{\varepsilon}\left(x_{1}-y\right) .
\end{aligned}
$$

By solving the equation $\bar{F} h\left(x, f_{\varepsilon}(x), \varepsilon\right)=0$ we see that $\bar{p}_{\varepsilon}=(0,0,0)$ is a tangency point of (8) for all $\varepsilon \neq 0$. Moreover, $\bar{F}^{2} h\left(\bar{p}_{\varepsilon}\right)=\theta$ and $\bar{G} h\left(\bar{p}_{\varepsilon}\right)=-\theta$. Thus, for each $\theta<0$ fixed, $\bar{p}_{\varepsilon}$ is a visible fold-regular singularity of (8). Therefore, the statement (ii) of Theorem A is not true.

2.1. Singular perturbation of fold-fold singularities. The next example shows that, in dimension two, fold-fold singularities are not persistent with respect to singular perturbations.

Example 2. Consider the following system

$$
\dot{x}=\left(\dot{x}_{1}, \dot{x}_{2}\right)=\left\{\begin{array}{ll}
\left(x_{2}, 1\right) & \text { if } x_{1}+\varepsilon \leq 0, \\
\left(x_{2}+\varepsilon,-1\right) & \text { if } x_{1}+\varepsilon \geq 0,
\end{array} \quad \varepsilon \dot{y}=y+2 \varepsilon\right.
$$

The reduced problem of (10) is given by

$$
\dot{x}=\left(\dot{x}_{1}, \dot{x}_{2}\right)=\left\{\begin{array}{ll}
\left(x_{2}, 1\right) & \text { if } x_{1} \leq 0, \\
\left(x_{2},-1\right) & \text { if } x_{1} \geq 0,
\end{array} \quad y=0 .\right.
$$

It is easy to see that $\bar{p}=(0,0)$ is a fold-fold singularity of (11). By denoting system (10) as in (5), we have that

$$
\bar{F} h=x_{2}, \quad \bar{F}^{2} h=1, \quad \bar{G} h=x_{2}+\varepsilon, \quad \bar{G}^{2} h=-1 .
$$

Thus, for all $\varepsilon \neq 0$, system (10) does not have fold-fold singularities in $\mathcal{M}_{\varepsilon}=\left\{x_{1}=-\varepsilon\right\}$. The point $(0,0,0)$ was unfolded by families $\bar{p}_{\varepsilon}=$ $(-\varepsilon, 0,-2 \varepsilon)$ and $\bar{q}_{\varepsilon}=(-\varepsilon,-\varepsilon,-2 \varepsilon)$ of fold singularities of the vector fields $\bar{F}=\left(x_{2}, 1, y / \varepsilon+\varepsilon\right)$ and $\bar{G}=\left(x_{2}+\varepsilon,-1, y / \varepsilon+\varepsilon\right)$, respectively.

On the other hand, in dimension greater than two, fold-fold singularities are persistent with respect to singular perturbations.

Theorem B. Let $Z_{\varepsilon}(x, y)$ be a $C^{r}$ family defined by (1), with $x \in \mathbb{R}^{n}$, $n \geq 3$ and $r \geq 2$. Consider $\bar{p}=\left(p, f_{0}(p), 0\right) \in \mathcal{S}_{0} \cap \mathcal{M}_{0}$ a fold-fold singularity of the reduced problem $Z_{0}$ and suppose that $Z_{\varepsilon}$ is locally simple at $\bar{p}$. Then there exists $\varepsilon_{1}>0$ such that: 
(i) There is a $C^{r-1}$ family $\left\{\bar{p}_{\varepsilon}: \varepsilon \in\left(-\varepsilon_{1}, \varepsilon_{1}\right)\right\}$ such that $\bar{p}_{0}=\bar{p}$ and $\bar{p}_{\varepsilon}$ is a fold-fold singularity of $Z_{\varepsilon}$.

(ii) If $\bar{p}$ is either an elliptic or parabolic or hyperbolic fold-fold of $Z_{0}$ then $\bar{p}_{\varepsilon}$ will be either an elliptic or parabolic or hyperbolic fold-fold of $Z_{\varepsilon}$, respectively.

Theorem B is proved in Section 5. It is illustrated by the example below.

Example 3. Consider the following system

$$
\dot{x}=\left\{\begin{array}{ll}
\left(x_{2}+x_{3}, 1,1\right) & \text { if } x_{1}+\varepsilon \leq 0, \\
\left(x_{2}-x_{3}+\varepsilon,-1,1\right) & \text { if } x_{1}+\varepsilon \geq 0,
\end{array} \quad \varepsilon \dot{y}=y+2 \varepsilon,\right.
$$

where $x=\left(x_{1}, x_{2}, x_{3}\right) \in \mathbb{R}^{3}$. The manifolds $\mathcal{M}_{\varepsilon}$ and $\mathcal{N}_{\varepsilon}$ are given, respectively, by $\mathcal{M}_{\varepsilon}=\left\{x_{1}=-\varepsilon\right\}$ and $\mathcal{N}_{\varepsilon}=\{y=-2 \varepsilon\}$. The critical manifold is $\mathcal{S}_{0}=\{y=0\}$. On $\mathcal{S}_{0}$ we have defined the reduced problem

$$
\dot{x}=\left(\dot{x}_{1}, \dot{x}_{2}, \dot{x}_{3}\right)=\left\{\begin{array}{ll}
\left(x_{2}+x_{3}, 1,1\right) & \text { if } x_{1} \leq 0, \\
\left(x_{2}-x_{3},-1,1\right) & \text { if } x_{1} \geq 0,
\end{array} \quad y=0 .\right.
$$

By denoting system (13) as in (4), we have that

$$
\widetilde{F} \widetilde{h}=x_{2}+x_{3}, \quad \widetilde{F}^{2} \widetilde{h}=2, \quad \widetilde{G} \widetilde{h}=x_{2}-x_{3}, \quad \widetilde{G}^{2} \widetilde{h}=-2 .
$$

Thus, $\bar{p}=(0,0,0)$ is an elliptic fold-fold singularity of (13). Now, let us write system (12) in the general form given by (5), where

$$
\bar{F}(x, y, \varepsilon)=\left(x_{2}+x_{3}, 1,1, \frac{y}{\varepsilon}+\varepsilon\right)
$$

and

$$
\bar{G}(x, y, \varepsilon)=\left(x_{2}-x_{3}+\varepsilon,-1,1, \frac{y}{\varepsilon}+\varepsilon\right) .
$$

We have that

$$
\bar{F} h=x_{2}+x_{3}, \quad \bar{F}^{2} h=2, \quad \bar{G} h=x_{2}-x_{3}+\varepsilon, \quad \bar{G}^{2} h=-2 .
$$

By solving the system $\bar{F} h\left(x, f_{\varepsilon}(x), \varepsilon\right)=0, \bar{G} h\left(x, f_{\varepsilon}(x), \varepsilon\right)=0$ we obtain the $\varepsilon$-family $\bar{p}_{\varepsilon}=\left(-\varepsilon,-\frac{\varepsilon}{2}, \frac{\varepsilon}{2},-2 \varepsilon\right)$. Since $\bar{F}^{2} h\left(\bar{p}_{\varepsilon}\right)=2$ and $\bar{G}^{2} h\left(\bar{p}_{\varepsilon}\right)=$ -2 , it follows that $\bar{p}_{\varepsilon}$ is an elliptic fold-fold singularity of (12), for all $\varepsilon \neq 0$ small.

For systems of the form (1) which are not locally simple at $\bar{p}$, we cannot guarantee the validity of the statement (ii) of Theorem B. 
Counter-example 2. Consider the Filippov slow-fast system in $\mathbb{R}^{4}$

$$
\dot{x}=\left\{\begin{array}{ll}
\left(x_{2}, 1,1\right) & \text { if } 2 y-x_{1} \leq 0, \\
\left(x_{2}-x_{3}+\varepsilon,-1,1\right) & \text { if } 2 y-x_{1} \geq 0,
\end{array} \quad \varepsilon \dot{y}=y-x_{1},\right.
$$

where $x=\left(x_{1}, x_{2}, x_{3}\right) \in \mathbb{R}^{3}$. For $\varepsilon=0$ in (14) we have the reduced problem

$$
\left(\dot{x}_{1}, \dot{x}_{2}, \dot{x}_{3}\right)=\left\{\begin{array}{ll}
\left(x_{2}, 1,1\right) & \text { if } x_{1} \leq 0, \\
\left(x_{2}-x_{3},-1,1\right) & \text { if } x_{1} \geq 0,
\end{array} \quad y=x_{1} .\right.
$$

It is easy to see that $\bar{p}=(0,0,0)$ is an elliptic fold-fold singularity of (15). Note that system (14) is not locally simple at $\bar{p}$.

By denoting system (14) as in (5), for $\varepsilon \neq 0$ we have

$$
\begin{aligned}
\bar{F} h & =-x_{2}+\frac{2}{\varepsilon}\left(y-x_{1}\right), & \bar{F}^{2} h & =-\frac{2}{\varepsilon} x_{2}-1+\frac{2}{\varepsilon^{2}}\left(y-x_{1}\right), \\
\bar{G} h & =-x_{2}+x_{3}-\varepsilon+\frac{2}{\varepsilon}\left(y-x_{1}\right), & \bar{G}^{2} h & =-\frac{2}{\varepsilon}\left(x_{2}-x_{3}+\varepsilon\right)+2+\frac{2}{\varepsilon^{2}}\left(y-x_{1}\right) .
\end{aligned}
$$

By solving the system $\bar{F} h\left(x, f_{\varepsilon}(x), \varepsilon\right)=0, \bar{G} h\left(x, f_{\varepsilon}(x), \varepsilon\right)=0$ we obtain the $\varepsilon$-family $\bar{p}_{\varepsilon}=(0,0, \varepsilon, 0)$. Since $\bar{F}^{2} h\left(\bar{p}_{\varepsilon}\right)=-1$ and $\bar{G}^{2} h\left(\bar{p}_{\varepsilon}\right)=2$, it follows that $\bar{p}_{\varepsilon}$ is a hyperbolic fold-fold singularity of (14), for all $\varepsilon \neq 0$ small. Therefore, the statement (ii) of Theorem B is not true.

\section{Singular perturbation of cusp singularities}

In this section we study the effect of singular perturbations at the singularities of the kind cusp of the reduced problem (4). We restrict our attention to the case $n=2$, that is, we consider families of systems of the form (1) where $x=\left(x_{1}, x_{2}\right) \in \mathbb{R}^{2}$ and $y \in \mathbb{R}$.

Definition 3.1. We say that $p \in \mathcal{M}$ is a cusp-regular singularity of (2) if

(i) $F h(p)=F^{2} h(p)=0, F^{3} h(p) \neq 0$ and $G h(p) \neq 0$; or

(ii) $G h(p)=G^{2} h(p)=0, G^{3} h(p) \neq 0$ and $F h(p) \neq 0$.

Unlike the fold-regular singularities, the cusp-regular ones are not persistent with respect to singular perturbations. In order to prove this statement, consider the following example.

Example 4. Consider the following Filippov slow-fast system in $\mathbb{R}^{3}$

$$
\dot{x}=\left(\dot{x}_{1}, \dot{x}_{2}\right)=\left\{\begin{array}{ll}
\left(-1,-x_{1}^{2}+\varepsilon\right) & \text { if } x_{2} \leq 0, \\
(1,1) & \text { if } x_{2} \geq 0,
\end{array} \quad \varepsilon \dot{y}=y .\right.
$$


The reduced problem of (16) is given by

$$
\dot{x}=\left(\dot{x}_{1}, \dot{x}_{2}\right)=\left\{\begin{array}{ll}
\left(-1,-x_{1}^{2}\right) & \text { if } x_{2} \leq 0, \\
(1,1) & \text { if } x_{2} \geq 0,
\end{array} \quad y=0 .\right.
$$

It is easy to see that $\bar{p}=(0,0)$ is a cusp-regular singularity of (17). By denoting system (16) as in (5), we have

$$
\bar{F} h=-x_{1}^{2}+\varepsilon, \quad \bar{F}^{2} h=2 x_{1}, \quad \bar{F}^{3} h=-2, \quad \bar{G} h=1 .
$$

Thus, for all $\varepsilon \neq 0$, the vector field $\bar{F}$ does not have cusp singularities in $\mathcal{M}_{\varepsilon}=\left\{x_{2}=0\right\}$. For $\varepsilon>0$, the cusp-regular point $(0,0)$ is unfolded by families $\bar{p}_{\varepsilon}=(\sqrt{\varepsilon}, 0,0)$ and $\bar{q}_{\varepsilon}=(-\sqrt{\varepsilon}, 0,0)$ of fold-regular singularities of (16). If $\varepsilon<0$, system (16) does not have tangency points on $\mathcal{M}_{\varepsilon}$.

We have the following result.

Theorem C. Let $Z_{\varepsilon}(x, y)$ be a $C^{r}$ family defined by (1), with $x \in \mathbb{R}^{2}$ and $r \geq 3$. Consider $\bar{p}=\left(p, f_{0}(p), 0\right) \in \mathcal{S}_{0} \cap \mathcal{M}_{0}$ a cusp-regular singularity of the reduced problem $Z_{0}$ with $\widetilde{F}(p) \widetilde{G}(p) \neq 0$ and suppose that $Z_{\varepsilon}$ is locally simple at $\bar{p}$. Then, there exist $\varepsilon_{0}>0$, a neighborhood $W$ of $\bar{p}$, and a $C^{r-1}$ function $g:\left(-\varepsilon_{0}, \varepsilon_{0}\right) \rightarrow \mathbb{R}$, such that:

(i) $g(\varepsilon)=0$ if and only if $Z_{\varepsilon}$ has a cusp-regular singularity at the point $\bar{p}_{\varepsilon}$.

(ii) If $g(\varepsilon) \neq 0$, there are two possibilities: If $g(\varepsilon)>0$, then $Z_{\varepsilon}$ does not have tangency points on $\mathcal{M}_{\varepsilon}$. If $g(\varepsilon)<0$, then $\bar{p}$ is unfolded by two distinct points $\bar{p}_{\varepsilon}$ and $\bar{q}_{\varepsilon}$ which are fold-regular singularities of $Z_{\varepsilon}$.

Theorem $\mathrm{C}$ is proved in Section 5. Note that we can choose $g(\varepsilon)=-\varepsilon$ in Example 4.

3.1. Singular perturbation of fold-cusp singularities. It is not difficult to show that fold-cusp singularities are not persistent with respect to singular perturbations in dimensions two and three. On the other hand, in dimension four they are persistent. In fact,

$$
\dot{x}=\left(\dot{x}_{1}, \dot{x}_{2}\right)=\left\{\begin{array}{ll}
\left(1, x_{1}\right) & \text { if } x_{2} \leq 0, \\
\left(-1,-x_{1}^{2}+\varepsilon\right) & \text { if } x_{2} \geq 0,
\end{array} \quad \varepsilon \dot{y}=y,\right.
$$

and

$$
\dot{x}=\left(\dot{x}_{1}, \dot{x}_{2}, \dot{x}_{3}\right)=\left\{\begin{array}{ll}
\left(1, x_{1}-x_{3},-1\right) & \text { if } x_{2} \leq 0, \\
\left(-1,-x_{1}^{2}+\varepsilon, 1\right) & \text { if } x_{2} \geq 0
\end{array} \quad \varepsilon \dot{y}=y\right.
$$

provide counter-examples for $n=2$ and $n=3$ respectively. 
Theorem D. Let $Z_{\varepsilon}(x, y)$ be a $C^{r}$ family defined by (1), with $x \in \mathbb{R}^{n}$, $n \geq 4$ and $r \geq 3$. Consider $\bar{p}=\left(p, f_{0}(p), 0\right) \in \mathcal{S}_{0} \cap \mathcal{M}_{0}$ a fold-cusp singularity of the reduced problem $Z_{0}$ and suppose that $Z_{\varepsilon}$ is locally simple at $\bar{p}$. Then there are $\varepsilon_{1}>0$ and a $C^{r-1}$ family $\left\{\bar{p}_{\varepsilon}: \varepsilon \in\left(-\varepsilon_{1}, \varepsilon_{1}\right)\right\}$ such that $\bar{p}_{0}=\bar{p}$ and $\bar{p}_{\varepsilon}$ is a fold-cusp singularity of $Z_{\varepsilon}$.

Theorem $\mathrm{D}$ is proved in Section 5. It is illustrated by the example below.

Example 5. Consider the following Filippov slow-fast system in $\mathbb{R}^{5}$

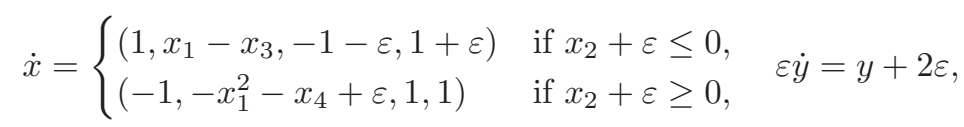

where $x=\left(x_{1}, x_{2}, x_{3}, x_{4}\right) \in \mathbb{R}^{4}$. The reduced problem associated to (18) is given by

$$
\dot{x}=\left\{\begin{array}{ll}
\left(1, x_{1}-x_{3},-1,1\right) & \text { if } x_{2} \leq 0, \\
\left(-1,-x_{1}^{2}-x_{4}, 1,1\right) & \text { if } x_{2} \geq 0,
\end{array} \quad y=0 .\right.
$$

By denoting system (19) as in (4), we have

$\widetilde{F} \widetilde{h}=x_{1}-x_{3}, \quad \widetilde{F}^{2} \widetilde{h}=2, \quad \widetilde{G} \widetilde{h}=-x_{1}^{2}-x_{4}, \quad \widetilde{G}^{2} \widetilde{h}=2 x_{1}-1, \quad \widetilde{G}^{3} \widetilde{h}=-2$. Thus, $\bar{p}=(1 / 2,0,1 / 2,-1 / 4,0)$ is a fold-cusp singularity of (19). Now, by denoting system (18) as in (5), we have

$$
\begin{array}{ll}
\bar{F} h=x_{1}-x_{3}, & \bar{F}^{2} h=2+\varepsilon, \\
\bar{G} h=-x_{1}^{2}-x_{4}+\varepsilon, & \bar{G}^{2} h=2 x_{1}-1, \quad \bar{G}^{3} h=-2 .
\end{array}
$$

By solving the system

$$
\begin{aligned}
& \bar{F} h\left(x, f_{\varepsilon}(x), \varepsilon\right)=0, \\
& \bar{G} h\left(x, f_{\varepsilon}(x), \varepsilon\right)=0, \\
& \bar{G}^{2} h\left(x, f_{\varepsilon}(x), \varepsilon\right)=0,
\end{aligned}
$$

we obtain the $\varepsilon$-family $\bar{p}_{\varepsilon}=(1 / 2,-\varepsilon, 1 / 2, \varepsilon-1 / 4,-2 \varepsilon)$. Since $\bar{F}^{2} h\left(\bar{p}_{\varepsilon}\right)=$ $2+\varepsilon$ and $\bar{G}^{3} h\left(\bar{p}_{\varepsilon}\right)=-2$, it follows that $\bar{p}_{\varepsilon}$ is a fold-cusp singularity of (18) for all $\varepsilon \neq 0$ small.

\section{Singular perturbation of hyperbolic singularities}

In this section we study the effect of singular perturbations at hyperbolic singularities of the reduced problem (4). We restrict our attention to the case $n=2$. 
Definition 4.1. We say that $p \in \mathcal{M}$ is a hyperbolic/equilibrium-regular singularity of $(2)$ provided $F(p)=0, G h(p) \neq 0$, and $D F(p)$ has eigenvalues $\lambda_{1}$ and $\lambda_{2}$ with nonzero real parts. Or, $G(p)=0, F h(p) \neq 0$ and $D G(p)$ has eigenvalues $\lambda_{1}$ and $\lambda_{2}$ with nonzero real parts.

Remark 3. We define hyperbolic/equilibrium-regular singularity for system (4) similarly to what was done in Definition 4.1. We note that system (4) is defined on the manifold $\mathcal{S}_{0}=\{H(x, y, 0)=0\}$.

We have the following result.

Theorem E. Let $Z_{\varepsilon}(x, y)$ be a $C^{r}$ family defined by $(1)$, with $x \in \mathbb{R}^{2}$ and $r>2$. Consider $\bar{p}=\left(p, f_{0}(p), 0\right) \in \mathcal{S}_{0} \cap \mathcal{M}_{0}$ a hyperbolic/equilibrium-regular singularity of the reduced problem $Z_{0}$. Suppose that $Z_{\varepsilon}$ is locally simple at $\bar{p}$ and that the eigenspaces of $Z_{0}$ are transverse to $\mathcal{M}_{0}$. Then, there exist $\varepsilon_{0}>0$, a neighborhood $W$ of $\bar{p}$ such that:

(i) Each $Z_{\varepsilon}=(\bar{F}, \bar{G})$, with $\varepsilon \in\left(-\varepsilon_{0}, \varepsilon_{0}\right)$, has a hyperbolic equilibrium point $\bar{p}_{\varepsilon} \in W$ such that $\bar{p}_{0}=\bar{p}$. Moreover, the hyperbolic equilibrium $\bar{p}_{\varepsilon}$ is of the same kind as $\bar{p}$.

(ii) If $\bar{p}_{\varepsilon} \notin \mathcal{M}_{\varepsilon} \cap W$, then there exists a family $\left\{\bar{q}_{\varepsilon}: \varepsilon \in\left(-\varepsilon_{0}, \varepsilon_{0}\right)\right\}$ of fold-regular singularities of $Z_{\varepsilon}$ such that $\bar{q}_{0}=\bar{p}$.

Theorem E is proved in Section 5. It is illustrated by the example below.

Example 6. Consider the following Filippov slow-fast system in $\mathbb{R}^{3}$

$$
\dot{x}=\left\{\begin{array}{ll}
(0,1) & \text { if } x_{1}+x_{2} \leq 0, \\
\left(x_{1},-x_{2}+\varepsilon\right) & \text { if } x_{1}+x_{2} \geq 0,
\end{array} \quad \varepsilon \dot{y}=y,\right.
$$

where $x=\left(x_{1}, x_{2}\right) \in \mathbb{R}^{2}$. For $\varepsilon=0$ in (20) we have the reduced problem

$$
\dot{x}=\left\{\begin{array}{ll}
(0,1) & \text { if } x_{1}+x_{2} \leq 0, \\
\left(x_{1},-x_{2}\right) & \text { if } x_{1}+x_{2} \geq 0,
\end{array} \quad y=0 .\right.
$$

It is easy to see that $\bar{p}=(0,0)$ is a saddle-regular singularity of $(21)$. For all $\varepsilon \neq 0$, the point $\bar{p}_{\varepsilon}=(0, \varepsilon, 0)$ is a saddle for the vector field $\bar{F}=\left(x_{1},-x_{2}+\varepsilon, y / \varepsilon\right)$, and note that $\bar{p}_{\varepsilon} \notin \mathcal{M}_{\varepsilon}=\left\{x_{1}+x_{2}=0\right\}$. Thus, system (20) does not have saddle-regular singularities on the switching manifold $\mathcal{M}_{\varepsilon}$. For $\varepsilon \neq 0$, there exists the family $\bar{q}_{\varepsilon}=(-\varepsilon / 2, \varepsilon / 2,0)$ of fold-regular singularities of (20). For $\varepsilon>0$, the point $\bar{q}_{\varepsilon}$ is an invisible fold for the vector field $\bar{F}=\left(x_{1},-x_{2}+\varepsilon, y / \varepsilon\right)$. If $\varepsilon<0$, then $\bar{q}_{\varepsilon}$ is a visible fold for $\bar{F}$ (see Figure 4 ). 

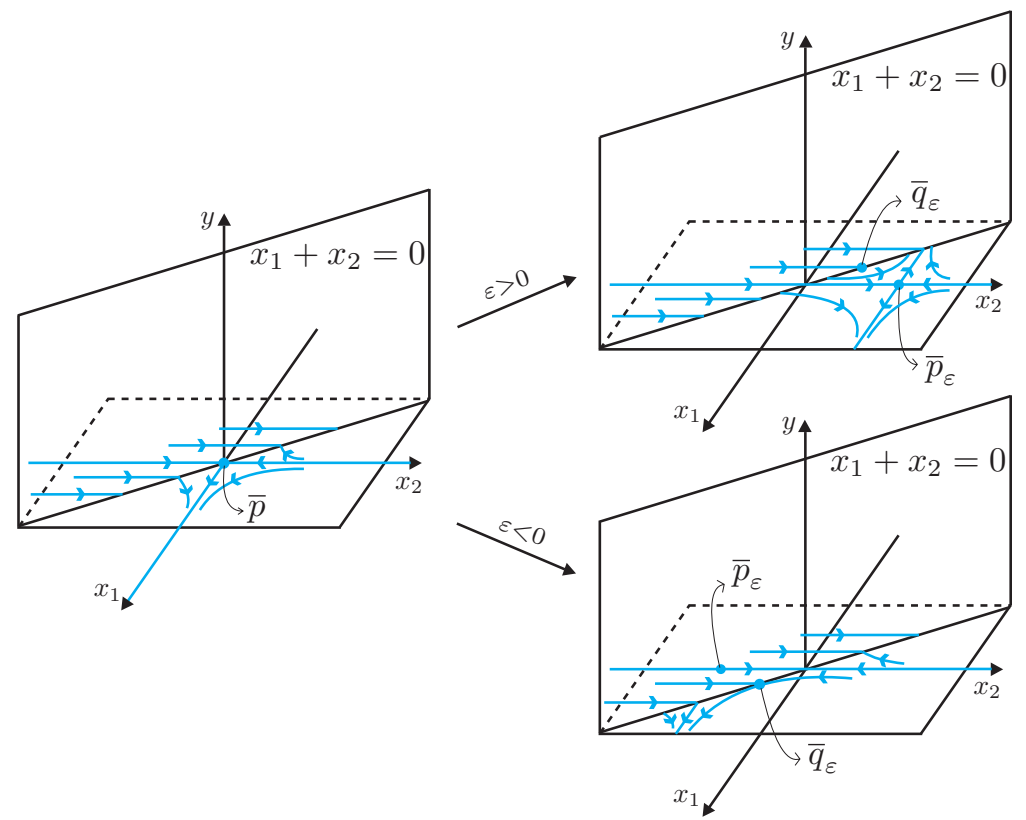

FiguRE 4. Unfolding of the saddle-regular singularity $\bar{p}$ of the reduced problem (21) of Example 6 .

\section{Proofs of Theorems A, B, C, D, and E}

In this section we prove Theorems A, B, C, D, and E. In all these proofs we will use the following result.

Lemma 5.1. Suppose that system (1) is locally simple at $\bar{p}=\left(p, f_{0}(p), 0\right) \in$ $\mathcal{S}_{0} \cap \mathcal{M}_{0}$. Then, for all $i \in \mathbb{N}$,

$$
\widetilde{F}^{i} \widetilde{h}(p)=F^{i} h\left(p, f_{0}(p), 0\right) \quad \text { and } \quad \widetilde{G}^{i} \widetilde{h}(p)=G^{i} h\left(p, f_{0}(p), 0\right) .
$$

Proof: Suppose that $i=1$. Since $\widetilde{F}(x)=F\left(x, f_{0}(x), 0\right)$ and $\widetilde{h}(x)=$ $h\left(x, f_{0}(x), 0\right)$, it follows by the Chain's rule that

$$
\widetilde{F} \widetilde{h}(p)=\frac{\partial \widetilde{h}}{\partial x}(p) \cdot F(\bar{p})=\left[\frac{\partial h}{\partial x} \cdot F+\frac{\partial h}{\partial y}\left(\nabla f_{0} \cdot F\right)\right](\bar{p}) .
$$

Since system (1) is locally simple at $\bar{p}$, one of the following conditions is satisfied: $\frac{\partial h}{\partial x}(\bar{p}) \neq 0$ and $h(p, y, 0)=0$, or $\frac{\partial H}{\partial x}(\bar{p})=0$. In the first case, by Remark 1 , it follows that $\frac{\partial h}{\partial y}(\bar{p})=0$. The second case implies $\nabla f_{0}(p)=0$. In both cases, the relation $(22)$ summarizes to $\widetilde{F} \widetilde{h}(p)=F h\left(p, f_{0}(p), 0\right)$. 
For $i=2$ we have

$$
\widetilde{F}^{2} \widetilde{h}(p)=\widetilde{F}(p)[\widetilde{F} \widetilde{h}(p)]=F\left(p, f_{0}(p), 0\right)\left[F h\left(p, f_{0}(p), 0\right)\right]=F^{2} h\left(p, f_{0}(p), 0\right) .
$$

By induction we can prove that $\widetilde{F}^{i} \widetilde{h}(p)=F^{i} h\left(p, f_{0}(p), 0\right)$, for all $i \in \mathbb{N}$.

The second relation $\widetilde{G}^{i} \widetilde{h}(p)=G^{i} h\left(p, f_{0}(p), 0\right)$ follows of similar way.

First we prove Theorem A.

Proof of Theorem A: We suppose that $\bar{p}=\left(p, f_{0}(p), 0\right)$ is a fold for the vector field $\widetilde{F}$ and regular for $\widetilde{G}$. The opposite case is similar. The fold-regular point $\bar{p}$ satisfies the following conditions

$$
\widetilde{F} \widetilde{h}(p)=0, \quad \widetilde{F}^{2} \widetilde{h}(p) \neq 0, \quad \text { and } \quad \widetilde{G} \widetilde{h}(p) \neq 0 .
$$

Since $Z_{\varepsilon}$ is locally simple at $\bar{p}$, by Lemma 5.1 , these conditions are equivalent to

$$
F h(\bar{p})=0, \quad F^{2} h(\bar{p}) \neq 0, \quad \text { and } \quad G h(\bar{p}) \neq 0 .
$$

On the other hand, for each $\varepsilon \neq 0$ small, the vector field $\bar{F}(x, y, \varepsilon)=$ $(F, H / \varepsilon)$ has a tangency at a point $q$ if, and only, if such point satisfies the equation $\bar{F} h(x, y, \varepsilon)=0$, that is equivalent to

$$
F h(x, y, \varepsilon)+\frac{\partial h}{\partial y} \frac{H}{\varepsilon}(x, y, \varepsilon)=0 .
$$

In order to obtain a family $\bar{p}_{\varepsilon}$ of tangency points such that $\bar{p}_{0}=\bar{p}$ we need to solve the equation (23) restricted to the manifold $y=f_{\varepsilon}(x)$. Since $H\left(x, f_{\varepsilon}(x), \varepsilon\right)=0$, it is enough to solve the following equation

$$
F h\left(x, f_{\varepsilon}(x), \varepsilon\right)=0 .
$$

We have $F^{2} h\left(p, f_{0}(p), 0\right) \neq 0$. But, $F^{2} h=F(F h)=\frac{\partial(F h)}{\partial x} \cdot F$. So, in particular, $\frac{\partial(F h)}{\partial x}\left(p, f_{0}(p), 0\right) \neq 0$. By the Implicit Function Theorem, for each $\varepsilon \neq 0$ sufficiently small there exists a unique $x=x(\varepsilon)$ such that $F h\left(x(\varepsilon), f_{\varepsilon}(x(\varepsilon)), \varepsilon\right)=0$. Take $\bar{p}_{\varepsilon}=\left(x(\varepsilon), f_{\varepsilon}(x(\varepsilon)), \varepsilon\right)$. Thus, $\bar{p}_{\varepsilon}$ is a family of tangency points of $Z_{\varepsilon}$ such that $\bar{p}_{0}=\bar{p}$.

For a fold-regular singularity of $Z_{\varepsilon}, \bar{p}_{\varepsilon}$, we need to prove that

$$
\left[F \cdot \frac{\partial}{\partial x}\left(\frac{\partial h}{\partial x} \cdot F+\frac{\partial h}{\partial y} \frac{H}{\varepsilon}\right)+\frac{H}{\varepsilon} \frac{\partial}{\partial y}\left(\frac{\partial h}{\partial x} \cdot F+\frac{\partial h}{\partial y} \frac{H}{\varepsilon}\right)\right]\left(\bar{p}_{\varepsilon}\right) \neq 0
$$

and

$$
\left(\frac{\partial h}{\partial x} \cdot G+\frac{\partial h}{\partial y} \frac{H}{\varepsilon}\right)\left(\bar{p}_{\varepsilon}\right) \neq 0 \text {. }
$$


Similarly as above since we are restricted to the manifold $y=f_{\varepsilon}(x)$ and $H\left(x, f_{\varepsilon}(x), \varepsilon\right)=0$, we obtain

$$
F^{2} h\left(\bar{p}_{\varepsilon}\right) \neq 0 \text { and } G h\left(\bar{p}_{\varepsilon}\right) \neq 0 .
$$

Since $\bar{p}=\left(p, f_{0}(p), 0\right)$ is a fold-regular singularity of $Z_{0}$ we have that $\widetilde{F}^{2} \widetilde{h}(p)=F^{2} h\left(p, f_{0}(p), 0\right) \neq 0$ and $\widetilde{G} \widetilde{h}(p)=G h\left(p, f_{0}(p), 0\right) \neq 0$. From the continuity of the functions $F^{2} h$ and $G h$, it follows that $F^{2} h(q) \neq 0$ and $G h(q) \neq 0$ for all $q$ in a given neighborhood of $\bar{p}$. In particular, for each $\varepsilon \neq 0$ sufficiently small, $F^{2} h\left(\bar{p}_{\varepsilon}\right) \neq 0$ and $G h\left(\bar{p}_{\varepsilon}\right) \neq 0$, i.e., $\bar{p}_{\varepsilon}$ is a fold-regular singularity of $Z_{\varepsilon}$.

In order to prove the statement (ii), suppose that the fold-regular $\bar{p}$ is visible. So, $\widetilde{F}^{2} \widetilde{h}(p)=F^{2} h\left(p, f_{0}(p), 0\right)<0$. Again, by continuity, we can conclude that $F^{2} h\left(\bar{p}_{\varepsilon}\right)<0$, for each $\varepsilon \neq 0$ sufficiently small, i.e., $\bar{p}_{\varepsilon}$ is a visible fold-regular singularity of $Z_{\varepsilon}$. Similarly, if $\bar{p}$ is an invisible fold-regular singularity of $Z_{0}$, then $\bar{p}_{\varepsilon}$ will also be an invisible fold-regular singularity of $Z_{\varepsilon}$.

Now we prove Theorem B.

Proof of Theorem B: The fold-fold singularity $\bar{p}$ of $Z_{0}$ satisfies the following conditions

$$
\widetilde{F} \widetilde{h}(p)=\widetilde{G} \widetilde{h}(p)=0, \quad \widetilde{F}^{2} \widetilde{h}(p) \neq 0, \quad \text { and } \quad \widetilde{G}^{2} \widetilde{h}(p) \neq 0 .
$$

Since $Z_{\varepsilon}$ is locally simple at $\bar{p}$, by Lemma 5.1 , these conditions are equivalent to

$$
F h(\bar{p})=G h(\bar{p})=0, \quad F^{2} h(\bar{p}) \neq 0, \quad \text { and } \quad G^{2} h(\bar{p}) \neq 0 .
$$

In order to obtain a family $\bar{p}_{\varepsilon}$ of tangency points of $\bar{F}$ and $\bar{G}$ such that $\bar{p}_{0}=\bar{p}$ we need to solve the system

$$
\begin{aligned}
& F h(x, y, \varepsilon)+\frac{\partial h}{\partial y} \frac{H}{\varepsilon}(x, y, \varepsilon)=0, \\
& G h(x, y, \varepsilon)+\frac{\partial h}{\partial y} \frac{H}{\varepsilon}(x, y, \varepsilon)=0,
\end{aligned}
$$

restricted to the manifold $y=f_{\varepsilon}(x)$. Since $H\left(x, f_{\varepsilon}(x), \varepsilon\right)=0$, it is enough to solve the following system

$$
\begin{aligned}
& F h\left(x, f_{\varepsilon}(x), \varepsilon\right)=0, \\
& G h\left(x, f_{\varepsilon}(x), \varepsilon\right)=0 .
\end{aligned}
$$

Let $\Sigma_{1}, \Sigma_{2}, \Sigma_{1}^{\varepsilon}$, and $\Sigma_{2}^{\varepsilon}$ be the $n$-dimensional manifolds in $\mathbb{R}^{n+1}$ given by $\left\{F h\left(x, f_{0}(x), 0\right)=0\right\},\left\{G h\left(x, f_{0}(x), 0\right)=0\right\},\left\{F h\left(x, f_{\varepsilon}(x), \varepsilon\right)=0\right\}$, 
and $\left\{G h\left(x, f_{\varepsilon}(x), \varepsilon\right)=0\right\}$, respectively. The fact that $\bar{p}$ is a tangency point of the vector fields $\widetilde{F}$ and $\widetilde{G}$ means that the manifolds $\mathcal{S}_{0}, \mathcal{M}_{0}$, $\Sigma_{1}$, and $\Sigma_{2}$ intersect transversally at $\bar{p}$. Since transversal intersection is a stable property, small perturbations of the manifolds $\mathcal{S}_{0}, \mathcal{M}_{0}, \Sigma_{1}$, and $\Sigma_{2}$ still produce a transversal intersection. So we can conclude that the manifolds $\mathcal{N}_{\varepsilon}, \mathcal{M}_{\varepsilon}, \Sigma_{1}^{\varepsilon}$, and $\Sigma_{2}^{\varepsilon}$ intersect transversally for all $\varepsilon \neq 0$ sufficiently small. Moreover, $\operatorname{dim}\left(\mathcal{N}_{\varepsilon} \cap \mathcal{M}_{\varepsilon} \cap \Sigma_{1}^{\varepsilon} \cap \Sigma_{2}^{\varepsilon}\right)=n-3$. This means that, for each $\varepsilon \neq 0$ sufficiently small, there exists a point $\bar{p}_{\varepsilon}$ such that $\bar{p}_{0}=\bar{p}$ and $\bar{p}_{\varepsilon}$ is a tangency point for the vector fields $\bar{F}=(F, H / \varepsilon)$ and $\bar{G}=(G, H / \varepsilon)$. Note that when $n=3$, the tangency point $\bar{p}_{\varepsilon}$ is isolated but if $n>3$ it is not.

In order to prove that $\bar{p}_{\varepsilon}$ is a fold-fold singularity of $Z_{\varepsilon}$ we need to prove that $F^{2} h\left(\bar{p}_{\varepsilon}\right) \neq 0$ and $G^{2} h\left(\bar{p}_{\varepsilon}\right) \neq 0$. Since $\bar{p}=\left(p, f_{0}(p), 0\right)$ is a foldfold singularity of $Z_{0}$ we have that $\widetilde{F}^{2} \widetilde{h}(p)=F^{2} h\left(p, f_{0}(p), 0\right) \neq 0$ and $\widetilde{G}^{2} \widetilde{h}(p)=G^{2} h\left(p, f_{0}(p), 0\right) \neq 0$. From the continuity of the functions $F^{2} h$ and $G^{2} h$, it follows that $F^{2} h(q) \neq 0$ and $G^{2} h(q) \neq 0$ for all $q$ in a given neighborhood of $\bar{p}$. In particular, for each $\varepsilon \neq 0$ sufficiently small, $F^{2} h\left(\bar{p}_{\varepsilon}\right) \neq 0$ and $G^{2} h\left(\bar{p}_{\varepsilon}\right) \neq 0$, i.e., $\bar{p}_{\varepsilon}$ is a fold-fold singularity of $Z_{\varepsilon}$.

In order to prove the statement (ii), suppose that the fold-fold $\bar{p}$ is elliptic. So, $\widetilde{F}^{2} \widetilde{h}(p)=F^{2} h\left(p, f_{0}(p), 0\right)>0$ and $\widetilde{G}^{2} \widetilde{h}(p)=G^{2} h\left(p, f_{0}(p), 0\right)<$ 0 . Again, by continuity, we can conclude that $F^{2} h\left(\bar{p}_{\varepsilon}\right)>0$ and $G^{2} h\left(\bar{p}_{\varepsilon}\right)<$ 0 , for each $\varepsilon \neq 0$ sufficiently small, i.e., $\bar{p}_{\varepsilon}$ is an elliptic fold-fold singularity of $Z_{\varepsilon}$. Similarly, if $\bar{p}$ is a parabolic (resp. hyperbolic) fold-fold of $Z_{0}$, then $\bar{p}_{\varepsilon}$ will be a parabolic (resp. hyperbolic) fold-fold of $Z_{\varepsilon}$.

Now we prove Theorem C.

Proof of Theorem $C$ : We suppose that $\bar{p}=\left(p, f_{0}(p), 0\right)$ is a cusp for the vector field $\widetilde{F}$ and regular for $\widetilde{G}$, i.e, $\widetilde{F} \widetilde{h}(p)=\widetilde{F}^{2} \widetilde{h}(p)=0, \widetilde{F}^{3} \widetilde{h}(p) \neq 0$, and $\widetilde{G} \widetilde{h}(p) \neq 0$. The opposite case is similar. Since $\widetilde{F}(p) \neq 0$ and $\widetilde{G}(p) \neq$ 0 we can choose $\varepsilon_{1}>0$ and a neighborhood $W$ of $\bar{p}$ in $\mathbb{R}^{3}$ such that $F(q) \neq 0$ and $G(q) \neq 0$, for all $q \in W$ and all $Z_{\varepsilon}=[(F, H / \varepsilon),(G, H / \varepsilon)]$ with $\varepsilon \in\left(-\varepsilon_{1}, \varepsilon_{1}\right)$. In order to prove statements (i) and (ii) of Theorem $\mathrm{C}$, we restrict our attention on the manifold $\mathcal{N}_{\varepsilon}=\{H(x, y, \varepsilon)=0\}$. We define $K:\left(-\varepsilon_{1}, \varepsilon_{1}\right) \times \mathbb{R} \rightarrow \mathbb{R}$ by

$$
K(\varepsilon, \alpha)=\left[F\left(r_{\varepsilon}(\alpha)\right) \wedge r_{\varepsilon}^{\prime}(\alpha)\right]\left[G\left(r_{\varepsilon}(\alpha)\right) \wedge r_{\varepsilon}^{\prime}(\alpha)\right]
$$

where $r_{\varepsilon}: \mathbb{R} \rightarrow \mathcal{M}_{\varepsilon}$ is an imbedding such that $r_{0}(0)=\bar{p}$, and " $\wedge$ " is the product $(a, b) \wedge(c, d)=a d-b c$, where $(a, b),(c, d) \in \mathbb{R}^{2}$. 


\section{Calling}

$$
\begin{aligned}
(I) & =\frac{d}{d \alpha}\left[F\left(r_{\varepsilon}(\alpha)\right)\right] \wedge r_{\varepsilon}^{\prime}(\alpha)-F\left(r_{\varepsilon}(\alpha)\right) \wedge r_{\varepsilon}^{\prime \prime}(\alpha), \\
(I I) & =\frac{d}{d \alpha}\left[G\left(r_{\varepsilon}(\alpha)\right)\right] \wedge r_{\varepsilon}^{\prime}(\alpha)-G\left(r_{\varepsilon}(\alpha)\right) \wedge r_{\varepsilon}^{\prime \prime}(\alpha), \\
(I I I) & =\frac{d^{2}}{d \alpha^{2}}\left[F\left(r_{\varepsilon}(\alpha)\right)\right] \wedge r_{\varepsilon}^{\prime}(\alpha)-2 \frac{d}{d \alpha}\left[F\left(r_{\varepsilon}(\alpha)\right)\right] \wedge r_{\varepsilon}^{\prime \prime}(\alpha)+F\left(r_{\varepsilon}(\alpha)\right) \wedge r_{\varepsilon}^{\prime \prime \prime}(\alpha), \\
(I V) & =\frac{d^{2}}{d \alpha^{2}}\left[G\left(r_{\varepsilon}(\alpha)\right)\right] \wedge r_{\varepsilon}^{\prime}(\alpha)-2 \frac{d}{d \alpha}\left[G\left(r_{\varepsilon}(\alpha)\right)\right] \wedge r_{\varepsilon}^{\prime \prime}(\alpha)+G\left(r_{\varepsilon}(\alpha)\right) \wedge r_{\varepsilon}^{\prime \prime \prime}(\alpha),
\end{aligned}
$$

we have that

$$
\frac{\partial K}{\partial \alpha}(\varepsilon, \alpha)=(I)\left[G\left(r_{\varepsilon}(\alpha)\right) \wedge r_{\varepsilon}^{\prime}(\alpha)\right]+(I I)\left[F\left(r_{\varepsilon}(\alpha)\right) \wedge r_{\varepsilon}^{\prime}(\alpha)\right]
$$

and

$$
\frac{\partial^{2} K}{\partial \alpha^{2}}(\varepsilon, \alpha)=(I I I)\left[G\left(r_{\varepsilon}(\alpha)\right) \wedge r_{\varepsilon}^{\prime}(\alpha)\right]+(I V)\left[F\left(r_{\varepsilon}(\alpha)\right) \wedge r_{\varepsilon}^{\prime}(\alpha)\right]+2(I)(I I) \text {. }
$$

By a direct calculation we obtain

$$
\frac{\partial K}{\partial \alpha}(0,0)=0 \quad \text { and } \quad \frac{\partial^{2} K}{\partial \alpha^{2}}(0,0) \neq 0 .
$$

In the above statements we used the fact that $\bar{p}$ is a cusp singularity of $\widetilde{F}$, regular for $\widetilde{G}$, and the hypothesis that $Z_{\varepsilon}$ is locally simple at $\bar{p}$ (Lemma 5.1 states that $F^{i} h(\bar{p})=\widetilde{F}^{i} \widetilde{h}(p)$ and $G^{i} h(\bar{p})=\widetilde{G}^{i} \widetilde{h}(p)$ ).

By the Implicit Function Theorem, there exist $0<\varepsilon_{0}<\varepsilon_{1}$, a neighborhood $J$ of 0 in $\mathbb{R}$, and a $C^{r-1}$ function $\xi:\left(-\varepsilon_{0}, \varepsilon_{0}\right) \rightarrow J$ such that $\frac{\partial K}{\partial \alpha}(\varepsilon, \xi(\varepsilon))=0$ and $\frac{\partial^{2} K}{\partial \alpha^{2}}(\varepsilon, \alpha) \neq 0$, for all $(\varepsilon, \alpha) \in\left(-\varepsilon_{0}, \varepsilon_{0}\right) \times J$. Assume for simplicity that $\frac{\partial^{2} K}{\partial \alpha^{2}}(0,0)>0$. The other case is similar. Choose $\varepsilon_{0}$ and $J$ such that $\frac{\partial^{2} K}{\partial \alpha^{2}}(\varepsilon, \alpha)>0$, for all $(\varepsilon, \alpha) \in\left(-\varepsilon_{0}, \varepsilon_{0}\right) \times J$.

For each $\varepsilon \in\left(-\varepsilon_{0}, \varepsilon_{0}\right)$, consider the function $f_{\varepsilon}: J \rightarrow \mathbb{R}$ given by $f_{\varepsilon}(\alpha)=K(\varepsilon, \alpha)$. Thus, for each $\varepsilon \in\left(-\varepsilon_{0}, \varepsilon_{0}\right), \xi(\varepsilon)$ is a minimum point of $f_{\varepsilon}$. Moreover:

(i) If $f_{\varepsilon}(\xi(\varepsilon))>0$ then $f_{\varepsilon}(\alpha)>0$, for all $\alpha \in J$. This means that $Z_{\varepsilon}$ is transverse to $\mathcal{M}_{\varepsilon}$ around $\bar{p}$. Thus, $Z_{\varepsilon}$ does not have tangency points on $\mathcal{M}_{\varepsilon}$.

(ii) If $f_{\varepsilon}(\xi(\varepsilon))=0$, then $f_{\varepsilon}(\alpha)=0$ if, and only if, $\alpha=\xi(\varepsilon)$. 
(iii) If $f_{\varepsilon}(\xi(\varepsilon))<0$ then by the Intermediate Value Theorem there exist $\alpha_{1}, \alpha_{2} \in J$, with $\alpha_{1}<\xi(\varepsilon)<\alpha_{2}$ such that $f_{\varepsilon}\left(\alpha_{1}\right)=f_{\varepsilon}\left(\alpha_{2}\right)=0$, however $\frac{\partial K}{\partial \alpha}\left(\varepsilon, \alpha_{i}\right) \neq 0, i=1,2$. This means that the contact of the vector field $\bar{F}$ with $\mathcal{M}_{\varepsilon}$ at $r_{\varepsilon}\left(\alpha_{1}\right)$ and $r_{\varepsilon}\left(\alpha_{2}\right)$ is quadratic and the vector field $\bar{G}$ is regular at $r_{\varepsilon}\left(\alpha_{1}\right)$ and $r_{\varepsilon}\left(\alpha_{2}\right)$. So $\bar{p}$ is unfolded by two distinct points $\left\{\bar{p}_{\varepsilon}=r_{\varepsilon}\left(\alpha_{1}\right)\right\}$ and $\left\{\bar{q}_{\varepsilon}=r_{\varepsilon}\left(\alpha_{2}\right)\right\}$ which are fold-regular singularities of $Z_{\varepsilon}$.

Define $g:\left(-\varepsilon_{0}, \varepsilon_{0}\right) \rightarrow \mathbb{R}$ by $g(\varepsilon)=K(\varepsilon, \xi(\varepsilon))$. This completes the proof of Theorem C.

Now we prove Theorem D.

Proof of Theorem D: We suppose that $\bar{p}=\left(p, f_{0}(p), 0\right)$ is a fold singularity for the vector field $\widetilde{F}$ and a cusp singularity for $\widetilde{G}$. The other case is similar. The fold-cusp singularity $\bar{p}$ of $Z_{0}$ satisfies the following conditions

$$
\widetilde{F} \widetilde{h}(p)=\widetilde{G} \widetilde{h}(p)=\widetilde{G}^{2} \widetilde{h}(p)=0, \quad \widetilde{F}^{2} \widetilde{h}(p) \neq 0, \quad \text { and } \quad \widetilde{G}^{3} \widetilde{h}(p) \neq 0 .
$$

Since $Z_{\varepsilon}$ is locally simple at $\bar{p}$, by Lemma 5.1 , these conditions are equivalent to

$$
F h(\bar{p})=G h(\bar{p})=G^{2} h(\bar{p})=0, \quad F^{2} h(\bar{p}) \neq 0, \quad \text { and } \quad G^{3} h(\bar{p}) \neq 0 .
$$

Let $\Sigma_{1}, \Sigma_{2}, \Sigma_{3}, \Sigma_{1}^{\varepsilon}, \Sigma_{2}^{\varepsilon}$, and $\Sigma_{3}^{\varepsilon}$ be the $n$-dimensional manifolds in $\mathbb{R}^{n+1}$ given by $\left\{F h\left(x, f_{0}(x), 0\right)=0\right\},\left\{G h\left(x, f_{0}(x), 0\right)=0\right\},\left\{G^{2} h\left(x, f_{0}(x), 0\right)=\right.$ $0\},\left\{F h\left(x, f_{\varepsilon}(x), \varepsilon\right)=0\right\},\left\{G h\left(x, f_{\varepsilon}(x), \varepsilon\right)=0\right\}$, and $\left\{G^{2} h\left(x, f_{\varepsilon}(x), \varepsilon\right)=\right.$ $0\}$, respectively. The fact that $\bar{p}$ is a fold-cusp singularity of $Z_{0}$ means that the manifolds $\mathcal{S}_{0}, \mathcal{M}_{0}, \Sigma_{1}, \Sigma_{2}$, and $\Sigma_{3}$ intersect transversally at $\bar{p}$ and that $F^{2} h(\bar{p}) \neq 0$ and $G^{3} h(\bar{p}) \neq 0$. Since transversal intersection is a stable property, we can conclude that the manifolds $\mathcal{N}_{\varepsilon}, \mathcal{M}_{\varepsilon}, \Sigma_{1}^{\varepsilon}, \Sigma_{2}^{\varepsilon}$, and $\Sigma_{3}^{\varepsilon}$ intersect transversally for all $\varepsilon \neq 0$ sufficiently small. Moreover, $\operatorname{dim}\left(\mathcal{N}_{\varepsilon} \cap \mathcal{M}_{\varepsilon} \cap \Sigma_{1}^{\varepsilon} \cap \Sigma_{2}^{\varepsilon} \cap \Sigma_{3}^{\varepsilon}\right)=n-4$. This means that, for each $\varepsilon \neq 0$ sufficiently small, there exists a point $\bar{p}_{\varepsilon} \in \mathcal{N}_{\varepsilon} \cap \mathcal{M}_{\varepsilon} \cap \Sigma_{1}^{\varepsilon} \cap \Sigma_{2}^{\varepsilon} \cap \Sigma_{3}^{\varepsilon}$. Clearly, $\bar{p}_{\varepsilon} \rightarrow \bar{p}$ when $\varepsilon \rightarrow 0$. By the continuity of the functions $F^{2} h$ and $G^{3} h$, we have that $F^{2} h\left(\bar{p}_{\varepsilon}\right) \neq 0$ and $G^{3} h\left(\bar{p}_{\varepsilon}\right) \neq 0$, for each $\varepsilon \neq 0$ sufficiently small. Therefore, $\bar{p}_{\varepsilon}$ is a fold-cusp singularity of $Z_{\varepsilon}$.

Note that when $n=4$ the fold-cusp point $\bar{p}_{\varepsilon}$ is isolated but if $n>4$ it is not.

We prove below Theorem E.

Proof of Theorem E: In order to prove statements (i) and (ii) of Theorem $\mathrm{E}$, we restrict our attention on the manifold $\mathcal{N}_{\varepsilon}=\{H(x, y, \varepsilon)=0\}$. 
We suppose that $\bar{p}$ is a hyperbolic equilibrium point for the vector field $\widetilde{F}$ and regular for $\widetilde{G}$. Thus, $\widetilde{F}(p)=F(\bar{p})=0, D \widetilde{F}(p)$ has eigenvalues $\lambda_{1}$ and $\lambda_{2}$ with nonzero real parts and $\widetilde{G} \widetilde{h}(p)=G h(\bar{p}) \neq 0$. Let $V$ be a neighborhood of $p$ in $\mathbb{R}^{2}$ such that $p$ is the unique singularity of $\widetilde{F}_{\left.\right|_{V}}$. It is known that there are $\varepsilon_{1}>0$ and a neighborhood $W$ of $\bar{p}$ in $\mathbb{R}^{3}$ such that each vector field $F(x, y, \varepsilon)$, with $\varepsilon \in\left(-\varepsilon_{1}, \varepsilon_{1}\right)$, has one unique hyperbolic singularity $\bar{p}_{\varepsilon}$ in $W$ and this singularity is of the same kind as $\bar{p}$.

First we consider the case where $\lambda_{1}$ and $\lambda_{2}$ are real eigenvalues with $\lambda_{1} \neq \lambda_{2}$. We can choose local coordinates $x=\left(x_{1}, x_{2}\right)$ around $p$ such that the components of $\widetilde{F}, \widetilde{F}_{1}$, and $\widetilde{F}_{2}$, satisfy

$$
\frac{\partial \widetilde{F}_{1}}{\partial x_{1}}(p)=\lambda_{1}, \quad \frac{\partial \widetilde{F}_{2}}{\partial x_{2}}(p)=\lambda_{2}, \quad \text { and } \quad \frac{\partial \widetilde{F}_{1}}{\partial x_{2}}(p)=\frac{\partial \widetilde{F}_{2}}{\partial x_{1}}(p)=0 .
$$

Define $K:\left(-\varepsilon_{1}, \varepsilon_{1}\right) \times \mathbb{R} \rightarrow \mathbb{R}$ by

$$
K(\varepsilon, \alpha)=F\left(r_{\varepsilon}(\alpha)\right) \wedge r_{\varepsilon}^{\prime}(\alpha),
$$

where $r_{\varepsilon}: \mathbb{R} \rightarrow \mathcal{M}_{\varepsilon}$ is an imbedding such that $r_{0}(0)=\bar{p}$, and " $\wedge$ " is the product $(a, b) \wedge(c, d)=a d-b c$, where $(a, b),(c, d) \in \mathbb{R}^{2}$.

Note that $K(0,0)=0$. If $r_{\varepsilon}(\alpha)=\left(r_{\varepsilon}^{1}(\alpha), r_{\varepsilon}^{2}(\alpha)\right)$, by hypothesis we have $\left(r_{0}^{1}\right)^{\prime}(0) \neq 0$ and $\left(r_{0}^{2}\right)^{\prime}(0) \neq 0$. Thus

$$
K(\varepsilon, \alpha)=F_{1}\left(r_{\varepsilon}(\alpha)\right) \wedge\left(r_{\varepsilon}^{2}\right)^{\prime}(\alpha)-F_{2}\left(r_{\varepsilon}(\alpha)\right) \wedge\left(r_{\varepsilon}^{1}\right)^{\prime}(\alpha)
$$

and, by direct computation, we obtain

$$
\frac{\partial K}{\partial \alpha}(0,0)=\left(r_{0}^{1}\right)^{\prime}(0)\left(r_{0}^{2}\right)^{\prime}(0)\left(\lambda_{1}-\lambda_{2}\right) .
$$

Since $\lambda_{1} \neq \lambda_{2}$, then $\frac{\partial K}{\partial \alpha}(0,0) \neq 0$. By the Implicit Function Theorem, there exist $0<\varepsilon_{0}<\varepsilon_{1}$, a neighborhood $J$ of 0 in $\mathbb{R}$ and a $C^{r-1}$ function $\xi:\left(-\varepsilon_{0}, \varepsilon_{0}\right) \rightarrow J$ such that $K(\varepsilon, \xi(\varepsilon))=0$ and $\frac{\partial K}{\partial \alpha}(\varepsilon, \alpha) \neq 0$, for all $(\varepsilon, \alpha) \in\left(-\varepsilon_{0}, \varepsilon_{0}\right) \times J$.

If $q_{\varepsilon}:=F\left(r_{\varepsilon}(\xi(\varepsilon))\right) \neq 0$, then this vector and $r_{\varepsilon}^{\prime}(\xi(\varepsilon))$ are linearly dependent because $K(\varepsilon, \xi(\varepsilon))=0$. Define $\bar{q}_{\varepsilon}=:\left(q_{\varepsilon}, f_{\varepsilon}\left(q_{\varepsilon}\right), \varepsilon\right)$. This means that $\left\{\bar{q}_{\varepsilon}: \varepsilon \in\left(-\varepsilon_{0}, \varepsilon_{0}\right)\right\}$ is a family of tangency points of $Z_{\varepsilon}$. Moreover, the condition $\frac{\partial K}{\partial \alpha}(\varepsilon, \xi(\varepsilon)) \neq 0$ imply that the contact of the vector field $\bar{F}$ with $\mathcal{M}_{\varepsilon}$ at $\bar{q}_{\varepsilon}$ is quadratic. 
Since $\widetilde{G} \widetilde{h}(p)=G h\left(p, f_{0}(p), 0\right) \neq 0$, from the continuity of the function $G h$, it follows that $G h\left(\bar{q}_{\varepsilon}\right) \neq 0$ for all $\varepsilon \neq 0$ sufficiently small, i.e., $\bar{q}_{\varepsilon}$ is regular for the vector field $\bar{G}$. This completes the proof in this case.

Now consider the case where $\lambda_{1}=\alpha+i \alpha_{1}$ and $\lambda_{2}=\beta+i \beta_{1}$ are complex eigenvalues with $\alpha \neq 0$ and $\beta \neq 0$. Let $x=\left(x_{1}, x_{2}\right)$ be a system of coordinates around $p$ such that

$$
\frac{\partial \widetilde{F}_{1}}{\partial x_{1}}(p)=\frac{\partial \widetilde{F}_{2}}{\partial x_{2}}(p)=\alpha \quad \text { and } \quad \frac{\partial \widetilde{F}_{1}}{\partial x_{2}}(p)=-\frac{\partial \widetilde{F}_{2}}{\partial x_{1}}(p)=\beta .
$$

Then, this case follows in a similar way as the first part.

\section{Closing remarks}

Non-smooth singularly perturbed systems were studied in this paper. We investigated the effect of singular perturbations at tangency points. We proved that singularities of the type fold are robust with respect to singular perturbations. On the other hand, cusp singularities are not robust with respect to singular perturbations. We also study the unfolding of cusp singularities and hyperbolic equilibria. In short we proved that:

- for any $n \geq 2$, fold-regular singularities are persistent;

- for $n=2$, cusp-regular singularities are not persistent;

- for $n=2$, fold-fold singularities are not persistent but for $n \geq 3$ they are;

- for $n=2,3$, fold-cusp singularities are not persistent but for $n \geq 4$ they are;

- for $n=2$, hyperbolic/equilibrium-regular singularities are not persistent.

Some open questions still remain. In [8], Sieber and Kowalczyk studied the robustness of periodic motion with sliding. They considered periodic orbits with an infinitesimally small sliding segment, that is, close to a grazing-sliding bifurcation, and proved that the local return map around the grazing periodic orbit develops a discontinuity if the condition on the existence of an attracting sliding region is violated. The question now is: If a sliding mode is persistent, can we develop a Fenichel's theory for this case?

Another open problem, similar to the one described in the previous paragraph, is the study of the robustness of the periodic motion with sewing with respect to singular perturbations. 


\section{Acknowledgments}

The first author is supported by FAPESP grant 2013/21947-6. The second and the third authors are partially supported by FAPESP, CAPES, CNPq and by the grant FP7-PEOPLE-2012-IRSES-316338. Also, the authors are very grateful to the referees for valuable comments and suggestions.

\section{References}

[1] B. Brogliato, "Nonsmooth impact mechanics", Models, dynamics and control, Lecture Notes in Control and Information Sciences 220, Springer-Verlag London, Ltd., London, 1996.

[2] P. T. Cardin, P. R. da Silva, and M. A. Teixeira, On singularly perturbed Filippov systems, European J. Appl. Math. 24(6) (2013), 835-856. DOI: 10.1017/S0956792513000211.

[3] M. Di Bernardo ET AL., Bifurcations in non-smooth dynamical systems, Publications of the Bristol Centre for Applied Nonlinear Mathematics 4 (2005).

[4] N. Fenichel, Geometric singular perturbation theory for ordinary differential equations, J. Differential Equations 31(1) (1979), 53-98. DOI: $10.1016 / 0022-0396(79) 90152-9$.

[5] A. F. FiLIPPOV, "Differential equations with discontinuous righthand sides", Translated from the Russian, Mathematics and its Applications (Soviet Series) 18, Kluwer Academic Publishers Group, Dordrecht, 1988.

[6] M. Kunze And T. KüPPER, Non-smooth dynamical systems: an overview, in: "Ergodic theory, analysis, and efficient simulation of dynamical systems", Springer, Berlin, 2001, pp. 431-452.

[7] Yu. A. Kuznetsov, S. Rinaldi, and A. Gragnani, One-parameter bifurcations in planar Filippov systems, Internat. J. Bifur. Chaos Appl. Sci. Engrg. 13(8) (2003), 2157-2188. DoI: 10.1142/ S0218127403007874.

[8] J. Sieber and P. KowalczyK, Small-scale instabilities in dynamical systems with sliding, Phys. D 239(1-2) (2010), 44-57. DOI: $10.1016 / j$.physd.2009.10.003.

[9] P. Szmolyan, Transversal heteroclinic and homoclinic orbits in singular perturbation problems, J. Differential Equations 92(2) (1991), 252-281. DOI: 10.1016/0022-0396(91) 90049-F. 
Pedro T. Cardin:

Departamento de Matemática

Faculdade de Engenharia de Ilha Solteira

UNESP - Univ Estadual Paulista

Rua Rio de Janeiro, 266

CEP 15385-000 Ilha Solteira, São Paulo

Brazil

E-mail address: pedrocardin@mat.feis.unesp.br

Paulo R. da Silva:

Departamento de Matemática

Instituto de Biociências, Letras e Ciências Exatas

UNESP - Univ Estadual Paulista

Rua C. Colombo, 2265

CEP 15054-000 S. J. Rio Preto, São Paulo

Brazil

E-mail address: prs@ibilce.unesp.br

Marco A. Teixeira:

IMECC-UNICAMP

CEP 13081-970, Campinas, São Paulo

Brazil

E-mail address: teixeira@ime.unicamp.br 